\title{
Role of Transesophageal Echocardiography in Retrieval of Dislodged Atrial Septal Defect Device Occluder
}

\author{
Minati Choudhury ${ }^{1}$ Vandana Bhardwaj ${ }^{1}$ Milind P. Hote ${ }^{1}$ \\ ${ }^{1}$ Cardiothoracic Sciences Centre, All India Institute of Medical \\ Sciences, New Delhi, India

\begin{abstract}
Address for Correspondence Dr. Minati Choudhury, MD, PG Dip, Professor, Department of Cardiac Anesthesiology, Cardiothoracic Sciences Centre, All India Institute of Medical Sciences, Ansari Nagar,
\end{abstract} \\ New Delhi 110029, India (e-mail: minati.2002@gmail.com).
}

J Card Crit Care:2020;4:144-146

\begin{abstract}
Keywords

- atrial septal defect

- amplatzer device

- transesophageal echocardiography

Transcatheter atrial septal defect (ASD) device closure has emerged as a safe and effective alternative procedure for surgical ASD repair. However, ASD device malposition and embolization has been reported with an incidence of 0.5 to $1.1 \%$. We report here a case of a 32-year-old male, who presented to the hospital 5 months after ASD device closure for routine follow-up. This case reports highlights the significance of transesophageal echocardiography (TEE) evaluation in successful surgical retrieval of a dislodged ASD Amplatzer device.
\end{abstract}

\section{Introduction}

Transcatheter atrial septal defect (ASD) device closure has emerged as a safe and effective alternative procedure for surgical ASD repair. ${ }^{1-3}$ However, ASD device malposition and embolization is a potentially life-threatening complication of this procedure. ${ }^{4}$ We report here that transesophageal echocardiographic (TEE) evaluation of a dislodged ASD device during surgical retrieval.

\section{Case Report}

We report here a case of a 32-year-old male, who presented to the hospital 5 months after ASD device closure for routine follow-up. The patient complained of nausea and dyspnea on exertion. Chest X-ray showed ASD device in right atrium (-Fig. 1). TTE revealed a displaced ASD device, which was 70\% located in the right atrium and $30 \%$ in the left atrium. The patient was taken for emergency surgical ASD device retrieval and ASD repair. The anesthesia induction and maintenance and monitoring were in accordance with the institutional protocol. TEE was performed using $x 7-2 t$ Philips ultrasound probe and ultrasound machine (Philips iE33 model, Bothell, WA, USA). The midesophageal four-chamber view revealed the dislodgement of ASD Amplatzer device, with three-fourth of the device located in the right atrium and the rest in the left atrium (-Fig. 2). The midesophageal, modified bicaval view also confirmed the dislodged device in the right atrium ( - Fig. 3). Bidirectional shunting of blood flow was seen across ASD. The displaced ASD device was not causing any obstruction of blood flow across mitral valve or tricuspid valve. The pulmonary venous flow as well as the systemic venous flow was found to be normal. The transgastric right ventricle inflow-outflow view revealed impingement of ASD device on the right atrial end of the inferior vena cava (IVC) ( - Fig. 4). However, the surgeon was able to put a two-stage venous cannula to commence cardiopulmonary bypass (CPB). The dislodged device was retrieved through the right atrium after undergoing normothermic CPB ( with Dacron patch closure. Post CPB, TEE evaluation revealed successful repair of ASD and normal flow across all four heart valves, pulmonary veins and systemic veins. The patient was extubated 5 hours after the procedure in the intensive care unit. The postextubation period was uneventful. published online October 8, 2020
DOI https://doi.org/ 10.1055/s-0040-1716945 ISSN 2457-0206. (c) 2020. Official Publication of The Simulation Society (TSS), accredited by International Society of Cardiovascular Ultrasound (ISCU).

This is an open access article published by Thieme under the terms of the Creative Commons Attribution-NonDerivative-NonCommercial-License, permitting copying and reproduction so long as the original work is given appropriate credit. Contents may not be used for commercial purposes, or adapted, remixed, transformed or built upon. (https://creativecommons.org/licenses/by-nc-nd/4.0/) Thieme Medical and Scientific Publishers Pvt. Ltd., A-12, 2nd Floor, Sector 2, Noida-201301 UP, India 


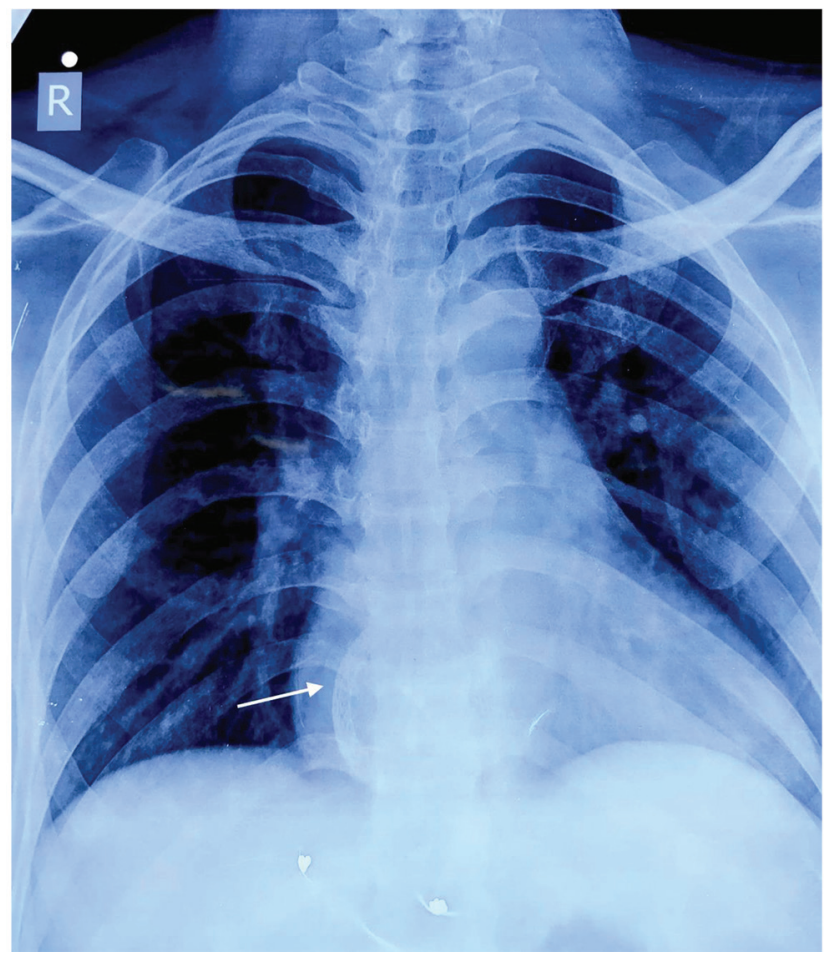

Fig. 1 Chest X-ray showing Amplatzer atrial septal defect occluder device (arrow) in right atrium.

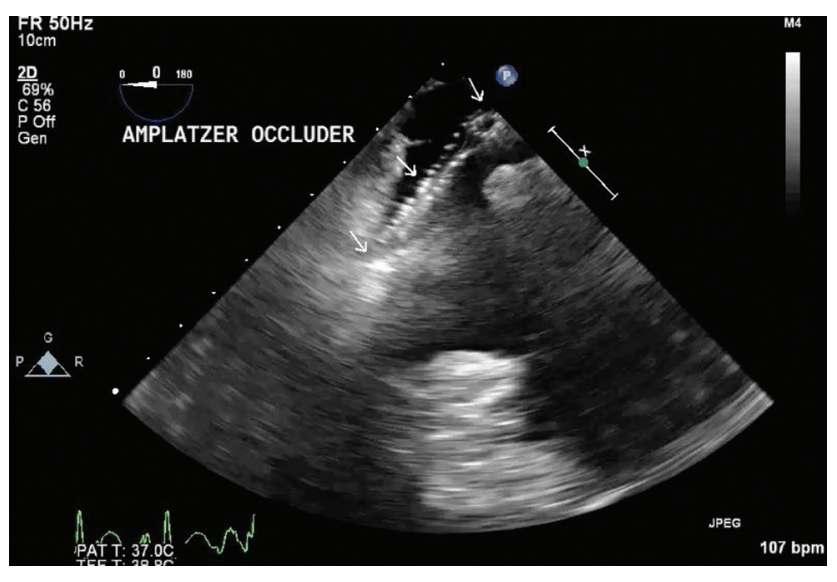

Fig. 2 Transesophageal echocardiography midesophageal four-chamber view (probe tilted to right), showing Amplatzer atrial septal device occluder in right atrium and atrial septal defect.

\section{Discussion}

ASD is one of the most common congenital heart diseases found in adults. Only ostium secundum type ASD is amenable to device closure. The transcatheter ASD device closure as well as intraoperative surgical device closure has been found to be safe and effective. The complication rate has been found to be lower and length of hospital stay has been found to be shorter with transcatheter device closure..$^{1-3}$ However, ASD device malposition and embolization has been reported with incidence of 0.5 to $1.1 \%{ }^{3,4}$ The risk factors for device

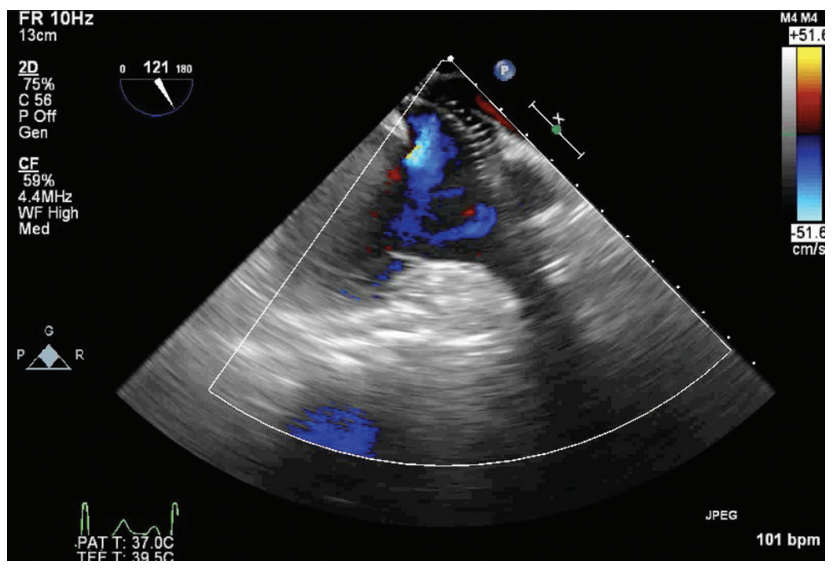

Fig. 3 Transesophageal echocardiography midesophageal modified bicaval view, showing dislodged atrial septal defect device occluder.

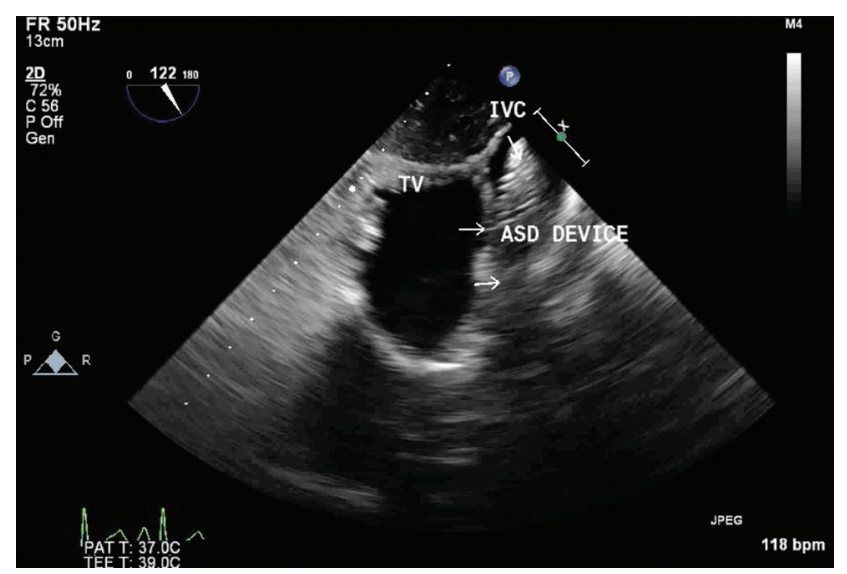

Fig. 4 Transesophageal echocardiography transgastric right ventricle inflow-outflow view, showing impingement of atrial septal defect (ASD) device occluder on IVC right atrium junction. Abbreviations: ASD, atrial septal defect; IVC, inferior vena cava.

embolization are large septal defects, larger devices and inadequate rims. The embolization of device most commonly occurs in the first few hours after implantation. However, late embolization up to 2 years after implantation has been reported..$^{5-8}$

In the present case, device dislodgement occurred 5 months after transcatheter ASD device implantation. The intraoperative TEE examination was used to guide surgical retrieval of the dislodged ASD device. TEE evaluation before CPB defined the location of dislodged ASD device and assessed the surrounding cardiac structures like heart valves, pulmonary veins, systemic veins and heart chambers. TEE was also utilized to help in the cannulation of right atrium, as the ASD device may get impinged on superior vena cava or IVC. In the present case, the device was impinging on the IVC end. However, a two-stage right atrium cannulation was successful. Finally, the TEE examination was performed to evaluate the ASD patch as well as the surrounding cardiac structure postsurgical repair. 


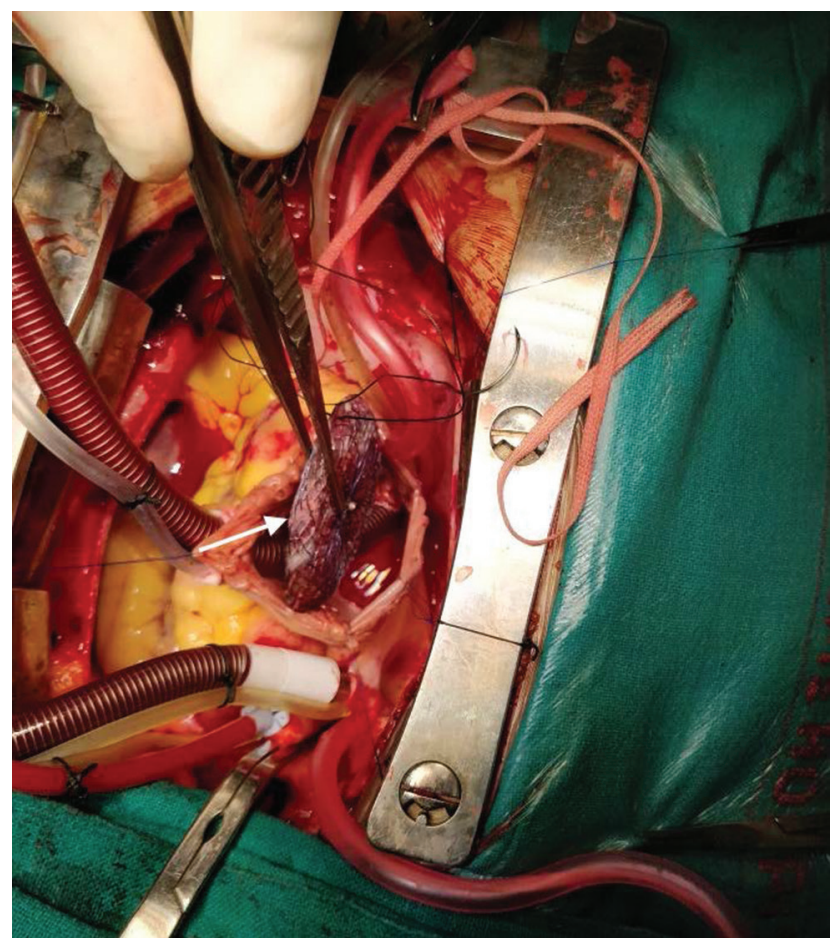

Fig. 5 Surgical retrieval of Amplatzer atrial septal defect device occluder (arrow).

\section{Conclusion}

This case reports highlights the significance of TEE evaluation in the successful surgical retrieval of a dislodged ASD Amplatzer device.

\section{Conflict of Interest}

None.

\section{References}

1 Moore J, Hegde S, El-Said H, et al; ACC IMPACT Steering Committee. Transcatheter device closure of atrial septal defects: a safety review. JACC Cardiovasc Interv 2013;6(5):433-442

2 Fischer G, Stieh J, Uebing A, Hoffmann U, Morf G, Kramer HH. Experience with transcatheter closure of secundum atrial septal defects using the Amplatzer septal occluder: a single centre study in 236 consecutive patients. Heart 2003;89(2):199-204

3 Du ZD, Hijazi ZM, Kleinman CS, Silverman NH, Larntz K; Amplatzer Investigators. Comparison between transcatheter and surgical closure of secundum atrial septal defect in children and adults: results of a multicenter nonrandomized trial. J Am Coll Cardiol 2002;39(11):1836-1844

4 Levi DS, Moore JW. Embolization and retrieval of the Amplatzer septal occluder. Catheter Cardiovasc Interv 2004;61(4): 543-547

5 Ussia GP, Abella R, Pome G, et al. Chronic embolization of an atrial septal occluder device: percutaneous or surgical retrieval? A case report. J Cardiovasc Med (Hagerstown) 2007;8(3):197-200

6 Chan KT, Cheng BC. Retrieval of an embolized amplatzer septal occluder. Catheter Cardiovasc Interv 2010;75(3):465-468

7 Errahmouni A, Hattaoui ME, Drighil A, Boumzebra D. Silent embolization of an Amplatzer septal occluder into the left ventricular outflow tract requiring emergent surgical retrieval. Ann Pediatr Cardiol 2012;5(1):89-91

8 Nath RK, Pandit N. Asymptomatic late embolization of Amplatzer septal occluder device. Indian Heart J 2017;69(3): 338-340 